\title{
Architectures and Services for Cultural Heritage Information
}

\author{
Organiser: Prof. Panos Constantopoulos \\ Institute of Computer Science (ICS) \\ Foundation for Research and Technology - Hellas (FORTH) \\ Science and Technology Park of Crete \\ GR - 71110 Heraklion, Crete, Greece \\ Tel: $+30-81-391634$ \\ Fax: $+30-81-391601$ \\ email: panos@ics.forth.gr
}

\begin{abstract}
The electronic processing of cultural heritage information involves stages of acquisition/production, storage, indexing, use/exploitation and transfer. The value of information is compounded by usage and association to other information. It is thus important to ensure the linking of information from disparate sources or held in different systems, access according to multiple viewpoints and needs, and exchange among different systems and user groups. Key issues are the selection of architectures that ensure interoperability and the development of services that support the recording, safeguarding, scientific study and promotion of cultural heritage, including artifacts and information sources. This panel will address these issues with a view towards the shaping of a DL domain.
\end{abstract}

Biographical note: Panos Constantopoulos is professor of Computer Science at the University of Crete and head of the Information Systems and Software Technology Division at the Institute of Computer Science, Foundation for Research and Technology-Hellas. His current research interests include multimedia information systems, conceptual modelling, information retrieval and decision support. 